\title{
The Management of Myelomeningocele Study: Short-Term Neonatal Outcomes
}

\author{
Natalie E. Rintoul ${ }^{\mathrm{a}}$ Roberta L. Keller ${ }^{\mathrm{b}}$ William F. Walsh ${ }^{\mathrm{c}}$ Pamela K. Burrows $^{\mathrm{d}}$ \\ Elizabeth A. Thom ${ }^{d}$ Michael J. Kallan ${ }^{\mathrm{e}}$ Lori J. Howell ${ }^{f} \quad$ N. Scott Adzick ${ }^{f}$ \\ for the MOMS Investigators
}

aDivision of Neonatology, Department of Pediatrics, Children's Hospital of Philadelphia, Philadelphia, PA, USA; ${ }^{b}$ Department of Pediatrics, Benioff Children's Hospital, University of California, San Francisco, San Francisco, CA, USA; 'Division of Neonatology, Vanderbilt University Medical Center, Nashville, TN, USA; ${ }^{\mathrm{d}}$ The Biostatistics Center, George Washington University, Washington, DC, USA; ${ }^{\circ}$ Department of Biostatistics, Epidemiology \& Informatics, Perlelman School of Medicine, University of Pennsylvania, Philadelphia, PA, USA; ${ }^{\mathrm{f} C e n t e r ~ f o r ~ F e t a l ~ D i a g n o s i s ~ a n d ~ T r e a t m e n t, ~}$ The Children's Hospital of Philadelphia, Philadelphia, PA, USA

\section{Keywords}

Myelomeningocele $\cdot$ Prenatal surgery · Preterm delivery · Neonatal outcome

\begin{abstract}
Introduction: The Management of Myelomeningocele Study was a multicenter randomized trial to compare prenatal and standard postnatal repair of myelomeningocele (MMC). Neonatal outcome data for 158 of the 183 randomized women were published in The New England Journal of Medicine in 2011. Objective: Neonatal outcomes for the complete trial cohort $(N=183)$ are presented outlining the similarities with the original report and describing the impact of gestational age as a mediator. Methods: Gestational age, neonatal characteristics at delivery, and outcomes including common complications of prematurity were assessed. Results: Analysis of the complete cohort confirmed the initial findings that prenatal surgery was associated with an increased risk for earlier gestational age at birth. Delivery occurred before 30 weeks of gestation in $11 \%$ of neonates that had fetal MMC repair. Adverse pulmonary sequelae were rare in the prenatal surgery group despite an increased rate of oligohydram-
\end{abstract}

nios. There was no significant difference in other complications of prematurity including patent ductus arteriosus, sepsis, necrotizing enterocolitis, periventricular leukomalacia, and intraventricular hemorrhage. Conclusion: The benefits of prenatal surgery outweigh the complications of prematurity.

C) 2020 S. Karger AG, Basel

\section{Introduction}

The National Institutes of Health-sponsored Management of Myelomeningocele Study (MOMS) compared the safety and efficacy of prenatal repair of myelomeningocele with that of standard postnatal repair. The trial was conducted by the National Institute of Child Health and Human Development (NICHD) Maternal-Fetal Surgery Units Group from 2003 to 2010. The trial was terminated prematurely due to a determination of efficacy by the

Presented as oral presentation at the 38th Annual Meeting of the International Fetal Medicine and Surgery Society, October 22-26, 2019, Sils, Switzerland.

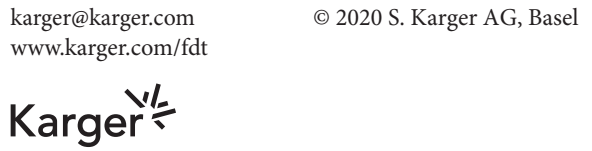


Data Safety Monitoring Committee, with publication of the results for the first 158 (86\%) of 183 women randomized [1]. Significant findings in that report included a decreased need for cerebrospinal fluid shunt at 12 months in the children that had undergone prenatal surgery before 26 weeks of gestation, with reduced hindbrain herniation and improved motor outcomes at 30 months in the prenatal surgery group. These children were more likely to be born preterm ( 79 vs. $15 \% ; p<0.001)$ [1]. The 1 -year neurosurgical outcomes for the complete cohort showed that prenatal surgery did not decrease the need for shunting in those fetuses with a cerebral ventricular size of $\geq 15 \mathrm{~mm}$ at initial screening [2]. Furthermore, the need for shunting was independent of lesion level and degree of hindbrain herniation. At 30 months of corrected age, neurological outcomes assessed in the full cohort confirmed that prenatal repair improves the primary outcome composite score of mental development and motor function [3].

The objective of the current report is to evaluate the neonatal outcomes of the MOMS trial for the full cohort, including morbidity and mortality. In particular, we wished to assess in more detail the consequences of early preterm delivery in those that underwent prenatal repair, to assist in counseling and informed consent prior to prenatal surgery.

\section{Methods}

\section{Study Population}

During the study period from 2003 to 2010 , a total of 183 mother-infant dyads were prospectively included in the study. A total of 91 were in the prenatal surgery group and 92 were in the postnatal surgery group. This study looked at the neonatal outcomes for the entire study cohort, beyond the original report, which included an additional 25 neonates [1].

Fetal inclusion criteria included a myelomeningocele located between $\mathrm{T} 1$ and S1, evidence of hindbrain herniation, a gestational age of 19-25 6/7 completed weeks at randomization, and a normal karyotype. Fetal exclusion criteria included a fetal anomaly unrelated to myelomeningocele and severe kyphosis. Women at increased risk for premature delivery were excluded.

Setting

Maternal-fetal dyads enrolled in the MOMS were randomized to 1 of 3 MOMS centers: Children's Hospital of Philadelphia (CHOP), Vanderbilt University Medical Center (VUMC), or the Benioff Children's Hospital, University of California San Francisco (UCSF). Neonatal data were submitted to an independent data coordinating center at the George Washington University and analyzed. The trial was approved by the institutional review board at each center.

\section{Management Protocols}

Prenatal Surgery Group: Experimental Care

Prenatal surgery occurred prior to $256 / 7$ weeks of gestation. In the event of preterm labor, one course of antenatal steroids was given between 24 and 34 weeks of gestation. Steroids were not given at the time of prenatal surgery. Delivery by cesarean section occurred in the event of preterm labor unresponsive to tocolysis, rupture of membranes at $\geq 34$ weeks of gestation, chorioamnionitis, placental abruption, nonreassuring fetal status, or if the patient reached 37 weeks' gestation. If the mother had rupture of membranes before 34 weeks' gestation, she was managed expectantly until planned delivery at 34 weeks by cesarean section.Postnatal Surgery Group: Standard Care

Delivery was by elective cesarean section at 37 weeks. Earlier delivery occurred for routine obstetric and fetal concerns. Repair of spina bifida by the same neurosurgical team as for the prenatal surgery occurred when the patient was determined to be clinically stable.

Delivery

All deliveries were by cesarean section during the trial period.

\section{Outcomes}

The final analysis of the complete cohort included 182 surviving neonates. General demographics included mortality, gestational age, birthweight, and Apgar scores.

Typical morbidities of prematurity including respiratory distress syndrome (RDS), surfactant use, apnea, hyperbilirubinemia, patent ductus arteriosus (PDA), sepsis, necrotizing enterocolitis (NEC), intraventricular hemorrhage (IVH), and periventricular leukomalacia (PVL) were assessed. Several neonatal outcomes were then stratified by gestational age at delivery in an effort to describe the impact of gestational age as a mediator. In addition, complications during repair (i.e., bradycardia), the incidence of pulmonary hypoplasia, pneumothorax, foot deformity, and length of stay were also assessed.

\section{Definition of Outcomes}

Prematurity was categorized by completed weeks of gestation as follows: $\geq 37$ weeks (term), 34-36 6/7 weeks (late preterm), 30$336 / 7$ weeks (preterm), and $<30$ weeks (very preterm). Perinatal death included a combination of fetal and neonatal deaths. Bradycardia during MMC repair was defined as heart rate below 110 beats per minute. An Apgar score $<7$ was defined as low when assigned at either 1 or $5 \mathrm{~min}$ of age.

Definition of the most common complications of prematurity included the following: RDS was defined as a clinical diagnosis of the RDS type I and the need for oxygen therapy (fraction of inspired oxygen, $\geq 0.40$ ) at $24 \mathrm{~h}$ of age or more; apnea was defined as a pause in breathing of $>20$ s or a pause in breathing accompanied by color change or bradycardia; PDA was reported only if the infant was treated with medications and/or surgery; sepsis was defined if bacteria isolated from blood culture, or urinary tract infection, meningitis, or pneumonia; NEC was defined as a confirmed clinical diagnosis with any of the following findings observed on radiography, at the time of surgery, or at autopsy: unequivocal presence of intramural air, intestinal perforation, erythema and induration of the abdominal wall, intra-abdominal abscess formation, or formation of a stricture after an episode of suspected NEC; IVH was reported as the most severe grade (I-IV), and PVL was reported if present (both unilateral and bilateral). 
Table 1. Fetal or neonatal outcomes

\begin{tabular}{|c|c|c|c|c|}
\hline Outcome: fetal or neonatal & $\begin{array}{l}\text { Prenatal surgery, } \\
n=91\end{array}$ & $\begin{array}{l}\text { Postnatal surgery, } \\
n=92\end{array}$ & $\begin{array}{l}\text { Relative risk } \\
(95 \% \mathrm{CI})\end{array}$ & $p$ value \\
\hline Bradycardia during fetal or neonatal repair, $n /$ total $n(\%)^{\mathrm{a}}$ & $9 / 90(10.0)$ & $0 / 92(0.0)$ & - & 0.001 \\
\hline Average gestational age at birth, weeks & $34.1( \pm 3.0)$ & $37.3( \pm 1.1)$ & & $<0.001$ \\
\hline \multicolumn{5}{|l|}{ Gestational age at birth category, $n /$ total $n(\%)$} \\
\hline$<30$ weeks & $10(11.0)$ & $0(0.0)$ & & \\
\hline$\geq 37$ weeks & $17(18.7)$ & $77(83.7)$ & & \\
\hline Birth weight mean, $g(S D)$ & $2,386(653)$ & $3,052(449)$ & & $<0.001$ \\
\hline Apgar score $1 \mathrm{~min}<7, n /$ total $n(\%)$ & 19/91 (20.9) & $14 / 92(15.2)$ & $1.37(0.74-2.56)$ & 0.34 \\
\hline Apgar score $5 \min <7, n /$ total $n(\%)$ & 10/91 (11.0) & $3 / 92(3.26)$ & $3.4(1.04-11.14)$ & 0.05 \\
\hline Foot deformity, $n /$ total $n(\%)$ & $45 / 90(50.0)$ & $41 / 92(44.6)$ & $1.12(0.82-1.53)$ & 0.55 \\
\hline Pneumothorax, $n /$ total $n(\%)$ & $2 / 89(2.3)$ & $1 / 92(1.1)$ & $2.07(0.19-22.40)$ & 0.62 \\
\hline Hyperbilirubinemia, $n /$ total $n(\%)$ & $40 / 89(44.9)$ & $11 / 92(12.0)$ & - & $<0.001$ \\
\hline PDA, $n /$ total $n(\%)$ & $3 / 90(3.3)$ & $0 / 92(0.0)$ & - & 0.12 \\
\hline Sepsis, $n /$ total $n(\%)$ & $4 / 90(4.4)$ & $1 / 92(1.1)$ & $4.09(0.47-35.88)$ & 0.12 \\
\hline NEC, $n /$ total $n(\%)$ & $1 / 90(1.1)$ & $0 / 92(0.0)$ & - & 0.49 \\
\hline IVH, $n /$ total $n(\%)$ & $11 / 89(12.4)$ & $3 / 92(3.3)$ & $3.79(1.18-12.39)$ & 0.03 \\
\hline PVL, $n /$ total $n(\%)$ & $4 / 89(4.5)$ & $2 / 92(2.2)$ & $2.07(0.39-11.01)$ & 0.44 \\
\hline Other intracranial hemorrhage (not IVH), $n /$ total $n$ (\%) & $2 / 89(2.2)$ & $2 / 92(2.1)$ & $1.03(0.07-15.41)$ & 1.00 \\
\hline Length of NICU stay average number of days (SD) & $19.2(19.3)$ & $12.9(7.0)$ & & 0.59 \\
\hline
\end{tabular}

PDA, patent ductus arteriosus; NEC, necrotizing enterocolitis; RSD, respiratory distress syndrome; IVH, intraventricular hemorrhage; PVL, periventricular leukomalacia. ${ }^{a}$ One mother randomized to the prenatal surgery group refused prenatal surgery. Bradycardia during repair was defined as heart rate below 110 beats per minute. Apnea was defined as a pause in breathing of $>20 \mathrm{~s}$. RDS was defined as a clinical diagnosis of the RDS type I and the need for oxygen therapy (fraction of inspired oxygen, $\geq 0.40$ ) at $24 \mathrm{~h}$ of age or more. PDA was reported if the infant was treated with medications or surgery. Sepsis was defined as confirmation on blood culture, confirmed urinary tract infection, meningitis, or pneumonia. NEC was defined as a confirmed clinical diagnosis with any of the following findings observed on radiography, at the time of surgery, or at autopsy: unequivocal presence of intramural air, perforation, erythema and induration of the abdominal wall, intra-abdominal abscess formation, or the formation of a stricture after an episode of suspected NEC.

\section{Statistical Analysis}

This updated analysis includes an additional 25 neonates and compares the prenatal versus postnatal repair groups according to the intention-to-treat principle. Of the 91 women assigned to the prenatal surgery group, 1 woman refused prenatal surgery after randomization. Relative risks and confidence intervals were calculated. Association between categorical variables was measured using Fisher's exact test, while for continuous values, we compared mean differences. Outcomes with a significant difference by surgery group and with sufficient numbers were also stratified by gestational age at birth. For all analyses, a nominal $p$ value of $<0.05$ was considered statistically significant.

\section{Results}

A total of 183 pregnancies were included, 91 in the prenatal surgery group and 92 in the postnatal surgery group. Baseline characteristics of the study population according to the surgical group are presented in Table 1. There were 2 deaths in each surgery group. In the prenatal surgery group, 1 of the 2 deaths was an intrauterine fetal demise at $262 / 7$ weeks' gestation and the other death occurred soon after delivery at $236 / 7$ weeks' gestation due to complications of prematurity. In the postnatal group, both patients died due to complications of severe Chiari II malformation.

The average gestational age at birth in the prenatal surgery group was 34.1 weeks and the postnatal surgery group was 37.3 weeks (Table 1). A total of $11.0 \%$ of prenatal surgery births occurred at $<30$ weeks, with no births at this low gestational age in the postnatal surgery group. In the prenatal surgery group, delivery during the planned early term (37 0/7-38 6/7 weeks') gestation window occurred in only $18.7 \%$ of the group; a total of $42.9 \%$ of the newborns were delivered late preterm (34 0/7-36 6/7 
Table 2. Neonatal outcomes stratified by gestational age at delivery

\begin{tabular}{|c|c|c|}
\hline Neonatal outcomes & $\begin{array}{l}\text { Prenatal } \\
\text { surgery }\end{array}$ & $\begin{array}{l}\text { Postnatal } \\
\text { surgery }\end{array}$ \\
\hline \multicolumn{3}{|l|}{ Birthweight } \\
\hline$<30$ weeks & $1,148.8(371.6)$ & - \\
\hline $30-336 / 7$ weeks & $2,039.6(304.6)$ & $1,604.0(446.9)$ \\
\hline $34-36$ 6/7 weeks & $2,639.9(351.3)$ & $2,804.2(317.6)$ \\
\hline$\geq 37$ weeks & $3,039.9(360.9)$ & $3,131.6(388.0)$ \\
\hline \multicolumn{3}{|c|}{ Apgar score $1 \mathrm{~min}<7, n /$ total $n(\%)$} \\
\hline$<30$ weeks & $6 / 10(60.0)$ & $0 / 0(-)$ \\
\hline $30-336 / 7$ weeks & $9 / 25(36.0)$ & $1 / 2(50.0)$ \\
\hline $34-36$ 6/7 weeks & $2 / 39(5.1)$ & $1 / 13(7.7)$ \\
\hline$\geq 37$ weeks & $2 / 17(11.8)$ & $12 / 77(15.6)$ \\
\hline \multicolumn{3}{|c|}{ Apgar score $5 \mathrm{~min}<7, n /$ total $n(\%)$} \\
\hline$<30$ weeks & $4 / 10(40.0)$ & $0 / 0(-)$ \\
\hline $30-336 / 7$ weeks & $5 / 25(20.0)$ & $1 / 2(50.0)$ \\
\hline $34-36$ 6/7 weeks & $1 / 39(2.6)$ & $1 / 13(7.7)$ \\
\hline$\geq 37$ weeks & $0 / 17(0.0)$ & $1 / 77(1.3)$ \\
\hline Foot deformity, $n /$ total $n(\%)$ & $45 / 90(50.0)$ & $41 / 92(44.6)$ \\
\hline$<30$ weeks & $5 / 9(55.6)$ & $0 / 0(-)$ \\
\hline 30-33 6/7 weeks & $11 / 25(44.0)$ & $1 / 2(50.0)$ \\
\hline 34-36 6/7 weeks & $18 / 39(46.2)$ & $7 / 13(53.8)$ \\
\hline$\geq 37$ weeks & $11 / 17(64.7)$ & $33 / 77(42.9)$ \\
\hline \multicolumn{3}{|l|}{ Apnea, $n /$ total $n(\%)$} \\
\hline$<30$ weeks & $8 / 8(100)$ & $0 / 0(-)$ \\
\hline 30-33 6/7 weeks & $16 / 25(64.0)$ & $1 / 2(50.0)$ \\
\hline 34-36 6/7 weeks & $10 / 39(25.6)$ & $7 / 13(53.8)$ \\
\hline$\geq 37$ weeks & $2 / 17(11.8)$ & $11 / 77(14.3)$ \\
\hline \multicolumn{3}{|l|}{ Surfactant, $n /$ total $n(\%)$} \\
\hline$<30$ weeks & $5 / 9(55.6)$ & $0 / 0(-)$ \\
\hline $30-336 / 7$ weeks & $7 / 25(28.0)$ & $0 / 2(0.0)$ \\
\hline $34-36$ 6/7 weeks & $5 / 39(12.8)$ & $4 / 12(33.3)$ \\
\hline$\geq 37$ weeks & $0 / 17(0.0)$ & $0 / 77(0.0)$ \\
\hline \multicolumn{3}{|l|}{$\mathrm{RDS}, n /$ total $n(\%)$} \\
\hline$<30$ weeks & $5 / 9(55.6)$ & $0 / 0(-)$ \\
\hline $30-336 / 7$ weeks & $12 / 25(48.0)$ & $0 / 2(0.0)$ \\
\hline 34-36 6/7 weeks & $3 / 39(7.7)$ & $3 / 13(23.1)$ \\
\hline$\geq 37$ weeks & $0 / 17(0.0)$ & $3 / 77(3.9)$ \\
\hline \multicolumn{3}{|c|}{ Hyperbilirubinemia, $n /$ total $n(\%)$} \\
\hline$<30$ weeks & $6 / 8(75.0)$ & $0 / 0(-)$ \\
\hline $30-336 / 7$ weeks & $18 / 25(72.0)$ & $0 / 2(0.0)$ \\
\hline 34-36 6/7 weeks & $14 / 39(35.9)$ & $3 / 13(23.1)$ \\
\hline$\geq 37$ weeks & $2 / 17(11.8)$ & 8/77 (10.4) \\
\hline \multicolumn{3}{|l|}{ IVH, $n /$ total $n(\%)$} \\
\hline$<30$ weeks & $2 / 8(25.0)$ & $0 / 0(-)$ \\
\hline $30-336 / 7$ weeks & $3 / 25(12.0)$ & $0 / 2(0.0)$ \\
\hline 34-36 6/7 weeks & $5 / 39(12.8)$ & $0 / 13(0.0)$ \\
\hline$\geq 37$ weeks & $1 / 17(5.9)$ & $3 / 77(3.9)$ \\
\hline
\end{tabular}

RSD, respiratory distress syndrome; IVH, intraventricular hemorrhage. weeks) and $38.5 \%$ of babies were delivered $<34$ weeks' gestation (Table 1). Of the 10 patients that delivered at $<30$ weeks' gestation, 3 delivered within 1 week of surgery (23-26 weeks). Significant differences were found between the prenatal and postnatal surgery groups with regard to gestational age at birth, birthweight, and incidence of RDS (Table 1). Birthweight was a mean of 2,386 $\pm 653 \mathrm{~g}$ in the prenatal group and 3,052 $\pm 449 \mathrm{~g}$ in the postnatal group $(p<0.001)$.

Neonatal outcomes stratified by gestational age at delivery are presented in Table 2. Low 1- or 5-min Apgar scores by gestational age group are shown in Table 2 . The increased incidence of RDS seen in the prenatal group was not related to sex. Of the 20 neonates with RDS, RDS was distributed almost evenly across sex (9 female, 11 male). As expected, the increased incidence of RDS was related to prematurity; in the prenatal surgery group, half of the newborns $<34$ weeks' gestation were diagnosed with RDS, while the incidence of RDS was similar for newborns $\geq 34$ weeks regardless of randomization groups (Table 2). There were no significant differences in the need for surfactant treatment within gestational age groups (Table 2). Rates of pulmonary hypoplasia and pneumothorax were low with no differences between the prenatal surgery and postnatal surgery groups (Table 1). There was also no significant difference in the incidence of clubfeet between the prenatal surgery and the postnatal surgery groups. There was a significant difference in hyperbilirubinemia treated with phototherapy seen in the prenatal surgery group $(p<0.001)$, which predominantly occurred in the premature babies (Table 2). There were no exchange transfusions. In the prenatal surgery group, 4 had sepsis; 2 were late infections with coagulase-negative staphylococci in premature infants $<30$ weeks, one was an Enterobacter urinary tract infection, and one was a pneumonia. In the postnatal group, one had meningitis (organism not reported). Overall, there was no significant difference in sepsis $(p=0.12)$ between the prenatal and postnatal surgery groups. There was also no difference in diagnosis of PVL $(p=0.44)$ or other ICH $(p=1.00)$ between the randomization groups.

The difference in IVH $(p=0.03)$ was mediated by prematurity. Among those born at term, 3 newborns had a diagnosis of IVH in the postnatal surgery group compared with 1 in the prenatal group (Tables 1, 2). Bradycardia during repair occurred in $10 \%$ of the prenatal surgery group $(n=9)$ and in none of the infants in the postnatal surgery group $(p<0.001$; Table 1$)$. Bradycardia was not associated with in utero fetal demise or delivery within 1 week of repair. One of the nine was noted to have 
PVL. None had grade 3 or 4 IVH as a neonate. Of note, neither of the 2 perinatal deaths in the prenatal surgery group had bradycardia during fetal repair.

PDA and NEC occurred with low frequency in the prenatal surgery group, and there were no significant differences with the postnatal surgery group. There was no difference in length of stay in the NICU between the 2 surgical groups (Table 1).

Apnea was significantly more common in the prenatal surgery group (RR 1.96, 1.22-3.14; $p=0.005$; Table 1 ). Among newborns that were mildly premature (34-36 weeks' gestation), apnea was more common in the postnatal surgery group (Table 2). Overall, severe hindbrain herniation was seen on the fetal MRI prior to randomization in $27 / 91$ (29.7\%) of the prenatal surgery group and $23 / 92(25.0 \%)$ of the postnatal surgery group.

In the patients with severe hindbrain herniation, follow-up MRI data were available on 23 of the 27 patients in the prenatal surgery group. Of these patients, $22 / 23$ (95.7\%) showed an improvement in hindbrain herniation, and in $4 / 23(17.4 \%)$, the herniation resolved completely.

No infants $(0 / 22)$ with severe hindbrain herniation prior to postnatal surgery had resolution of hindbrain herniation on follow-up MRI. Of the patients with mild/ moderate herniation, follow-up MRI data were available on 60 of the 64 patients in the prenatal surgery group. Of these patients, $3 / 60$ (5.0\%) worsened and 35/60 (58.3\%) remained unchanged, and in 22/60 (36.7\%), the herniation resolved completely. Follow-up MRI data were available on 64 of the 69 patients in the postnatal surgery group with mild/moderate herniation on the fetal MRI prior to randomization. Of these patients, 13/64 (20.3\%) worsened and $47 / 64(73.4 \%)$ were unchanged, and in 4/64 (6.3\%), hindbrain herniation resolved completely. In summary, fewer infants with mild/moderate hindbrain herniation on fetal MRI prior to randomization worsened after prenatal surgery, and more had complete resolution compared with the infants in the postnatal surgery group.

Treatment for apnea in the neonatal period was required in $0 / 4$ infants with complete resolution of severe hindbrain herniation and 7/18 (38.9\%) with improved hindbrain herniation in the prenatal surgery group. All 7 infants requiring treatment were premature $(<37$ weeks' gestation). For infants in the postnatal surgery group with severe hindbrain herniation prior to postnatal surgery, $4 / 22(18.2 \%)$ required treatment for apnea in the neonatal period. Three of the 4 in this group were term ( $\geq 37$ weeks' gestation).

Management of Myelomeningocele Study: Short-Term Neonatal Outcomes

\section{Discussion}

The MOMS was a multicenter randomized trial to compare prenatal and standard postnatal repair of myelomeningocele [1]. This study established the clinical benchmark for fetal myelomeningocele repair. The Committee on Obstetric Practice recommends that women carrying a fetus with a myelomeningocele should be made aware of the MOMS trial findings and be counseled regarding the option of maternal-fetal surgery in an experienced center, including both the risks and benefits to the woman and the baby $[4,5]$. Neural tube defects are the second most common major congenital anomaly after cardiac malformations [6]. It is important for neonatologists and other specialists to understand the findings in this study to counsel an increasing number of such patients that will be expected to consider fetal surgery for myelomeningocele in the future. The goal of this secondary analysis of the complete MOMS neonatal cohort was twofold: first, to further assess the neonatal outcomes and risks for prematurity in the prenatal surgery group, thereby providing additional information for general counseling to improve informed consent; and second, to describe the impact of gestational age on outcomes.

In the original trial report, significant differences were found between the prenatal and postnatal surgery groups with regard to gestational age at birth, birthweight, and RDS (without accounting for gestational age). We found that in the complete cohort, $11 \%$ that underwent prenatal surgery delivered at $<30$ weeks. The proportion previously reported was $13 \%$ [1]. Of note, almost $2 / 3$ (61.5\%) delivered at $\geq 34$ weeks' gestation. Preterm labor is a known, serious complication of prenatal surgery [7]. The maternal morbidity was reported for the whole cohort in the MOMS study by Johnson et al. [8]. Prenatal surgical intervention was associated with higher pregnancy complications that may lead to preterm delivery, including oligohydramnios, chorioamniotic separation, and premature membrane rupture $(p<0.001)$ [8]. Oligohydramnios occurred in $20 \%$ of the prenatal surgery group in the complete cohort [8]. In a multivariate analysis, oligohydramnios was also associated with an increase in odds of $>9$ fold for delivery $<34$ weeks 0 days [8]. Oligohydramnios is a known risk factor for pulmonary hypoplasia in the neonate. Although the specific mechanism is unknown, low amniotic fluid volume creates a restrictive environment in utero which may impact thoracic and fetal lung growth and lead to pulmonary hypoplasia [9]. Despite a significant difference in the rate of oligohydramnios in the prenatal surgery group, pulmonary hypoplasia and

Fetal Diagn Ther 2020;47:865-872 
pneumothorax were rare in the prenatal surgery group (1 and $2 \%$, respectively; Table 1 ). Furthermore, there was not an increased incidence of clubfeet due to in utero constraint in the oligohydramnios group. Despite an increased rate of premature rupture of membranes, prenatal surgery did not confer an increased risk of neonatal infection. Furthermore, the postnatal infections in the prenatal surgery group were typical of organisms seen in late-onset neonatal sepsis in preterm neonates that have not undergone fetal surgery.

The significant difference in overall birthweight observed between the prenatal group and the postnatal group was consistent with what was previously reported [1]. This difference was influenced by the contribution of low-birthweight infants in the prenatal surgery group. There was a significant difference in RDS between the prenatal and postnatal surgery groups consistent with what was previously reported [1]. In the current analysis, lower gestational age was found to be an important mediator of this difference, with no significant difference shown between the late preterm and early term groups. Furthermore, the incidence of RDS in the $\geq 37$ weeks' newborns in the postnatal surgery group (3.9\%) was similar to a previously published rate of RDS (3.7\%) documented for elective repeat cesarean section at 37 weeks, with no RDS reported in the prenatal surgery group in our study [10]. Another publication reported similar proportions of a combined incidence of RDS and transient tachypnea of the newborn after caesarean section of 33\% at 34 weeks, $22 \%$ at 35 weeks, $17 \%$ at 36 weeks, $5 \%$ at 37 weeks, and $2 \%$ at 38 weeks [11]. Our data on RDS alone in the prenatal surgery group, therefore, are somewhat reassuring as the proportion of affected infants in this closely monitored group is equal to or lower than published data.

There was a significant difference in apnea between the entire prenatal and postnatal surgery groups. This difference was not significant in the subgroup analysis between the late preterm and early term age groups and was likely mediated by the much larger proportion of infants below 34 weeks in the prenatal surgery group earlier gestational age. While we found that the incidence of apnea decreased with increasing gestational age, overall rates were higher than those previously published for babies without myelomeningocele at similar gestational ages. In the late preterm group, apnea was recorded in $26 \%$ in the prenatal surgery group and 54\% of the postnatal surgery group, higher in both groups compared with $20 \%$ previously described in healthy newborns at the same gestational age [12]. Similarly, the $11.8 \%$ incidence of apnea in infants with MMC prenatally repaired and born at term (>37 weeks' gestation) is significantly higher than the $0.1 \%$ incidence reported in healthy term infants without MMC $[13,14]$. Apnea is typically thought to be related to symptomatic Chiari II malformations with hindbrain herniation in infants with myelomeningocele. Interestingly, none of the infants with complete resolution of severe hindbrain herniation had apnea in the neonatal period. Furthermore, apnea requiring treatment was seen in the premature infants in the prenatal surgery group.

The most common severe complications of prematurity include RDS, bronchopulmonary dysplasia, PDA, sepsis, NEC, IVH, and retinopathy of prematurity [15]. This study of the complete cohort showed no significant difference in the short-term complications of prematurity in the prenatal surgery group as compared with the postnatal surgery group including PDA, sepsis, NEC, IVH, or PVL.

Of note, we did not find an association with an increased risk of preterm delivery and short-term adverse neurologic sequelae in those infants that experienced bradycardia during prenatal repair. Furthermore, recent reports have shown a decreased incidence of fetal bradycardia during repair. One center reported a $5 \%$ incidence compared with the $10 \%$ documented in the MOMS Trial [16].

Others have shown that the complications of prematurity following prenatal surgery for myelomeningocele are similar to those of prematurity secondary to other causes [17]. Hyperbilirubinemia treated with phototherapy was significantly more common in the prenatal surgery group. However, this significance was no longer evident with comparison of the late preterm and early term groups. In fact, the incidence of hyperbilirubinemia was similar to reports of near-term infants in whom $25 \%$ are treated with phototherapy and $10 \%$ in term infants $[18,19]$.

It is important to note that $43 \%$ of the prenatal surgery group delivered in the vulnerable late preterm gestation, 34-36 6/7 weeks' gestation. Late preterm births account for three quarters of all preterm births [20]. Children in this age group have been shown to have increased neurodevelopmental morbidity after discharge [21]. While it is reassuring that there were no significant short-term differences in outcomes in the late preterm group found in this review, the results of the 6- to 10-year follow-up of these children will be important. Other neonatal neurologic and urologic data from these infants have been reported previously $[2,22]$.

Recent surveys of physicians regarding their views on prenatal myelomeningocele repair have been positive. In a multivariable analysis, neonatologists were more likely 
to report prenatal repair as "very favorable" (OR 1.6; 95\% CI: 1.03-2.5) [23]. In a physician survey that examined physician views regarding the benefits and burdens of prenatal surgery for MMC, most physicians agreed that denying the benefits of open maternal-fetal surgery is unfair to the future child [24]. However, most would recommend a hypothetical fetoscopic technique if associated with decreased maternal morbidity. A survey of physicians showed that neonatologists were more likely to be in the fetocentric group with regard to their attitude to fetal surgery. Neonatologists ranked the relative importance of maternal-fetal surgery considerations in terms of neonatal benefits, risk of fetal death, and risk of maternal complications above the risk of prematurity [25].

The North American Fetal Therapy Network (NAFTNet) involves a growing number of centers performing fetal MMC repair as part of a Fetal MMC Repair Consortium. In addition, new centers worldwide are offering fetal surgery for MMC. Following the original New England Journal of Medicine report, centers are gaining experience and refining the prenatal management. Details such as the timing of fetal surgery have been found to affect prematurity as well. Soni et al. [26] showed that fetal surgery performed at $\geq 23$ weeks gestation is associated with a marked reduction in chorioamniotic separation and preterm premature rupture of membranes resulting in decreased preterm deliveries. Others are developing modified surgical techniques and reporting decreased premature rupture of membranes and chorioamniotic membrane separation [27, 28]. Experience and attention to detail are of the utmost importance to improving the outcomes in patients with myelomeningocele.

\section{Conclusion}

The advantages of prenatal surgery for myelomeningocele including decreased risk of death or need for shunting by the age of 12 months, decreased hindbrain herniation, and improved motor function must be balanced against the disadvantages, including the risk of prematurity. Analysis of the complete cohort confirmed the initial preliminary findings [1]. Gestational age was a significant mediator, and interestingly, prematurity did not seem to result in substantial or significant morbidity. Furthermore, oligohydramnios following prenatal surgery was not found to be associated with adverse pulmonary sequelae in the newborns. Parents must be counseled regarding known benefits and risks in order to make informed consent regarding prenatal surgery which is now a standard of care option.

\section{Acknowledgements}

Other participants in the MOMS trial were as follows: The Children's Hospital of Philadelphia, Pennsylvania: Alan Flake, MD; Holly Hedrick, MD; Leslie N. Sutton, MD; Jamie Koh, RN, MSN; Jack Rychik, MD; Mark P. Johnson, MD; Julie Moldenhauer, MD; R. Douglas Wilson, MD; Beverly Coleman, MD; David Cohen, MD; Patrick Pasquariello, MD; and Larissa Bilaniuk, MD. University of California, San Francisco, California: Michael Harrison, MD; Hanmin Lee, MD; Nalin Gupta, MD, PhD; Diana L. Farmer, MD; Jody Farrell, RN, MSN; Tamara Ryan, RN; and Rachel Perry, RN. Vanderbilt University Medical Center, Nashville, Tennessee: John Brock III, MD; Mary Dabrowiak, RN, MSN; Katharine Wenstrom, MD; Joseph Bruner, MD; Noel B. Tulipan, MD; Edmund Yang, MD, PhD; Nancy Chescheir, MD; and Tracy Perry. Columbia University College of Physicians and Surgeons, New York: Mary E. D'Alton, MD; The Biostatistics Center, George Washington University, Washington, DC: Erin Greenbaum Musok, MA; and Kristen Holloway, MS. The Eunice Kennedy Shriver National Institute of Child Health and Human Development, Bethesda, Maryland: Catherine Y. Spong, MD; and Rosemary Higgins, MD. The authors thank Anne Ades, MD, for comments on the manuscript.

\section{Statement of Ethics}

This research complies with the guidelines for human studies and was conducted ethically in accordance with the World Medical Association Declaration of Helsinki. This trial was approved by the Eunice Kennedy Shriver National Institute of Child Health and Human Development and by the National Institutes of Health. The study protocol was approved by each institution's committee on human research. In those electing to participate in the study, written informed consent was obtained.

\section{Conflict of Interest Statement}

The authors have no conflicts of interest and no financial relationships relevant to this article to disclose.

\section{Funding Sources}

All phases of this study were funded by the National Institutes of Health (NIH).

\section{Author Contributions}

Dr. Rintoul conceptualized and designed the substudy together with the other coauthors, performed part of the analyses, and drafted the initial manuscript. Dr. Keller and Dr. Walsh were involved in conceptualizing and designing the substudy and critically revising the manuscript and reviewing the statistical analyses. Ms. Burrows and Dr. Thom contributed to critical aspects of the conduct of this research including monitoring of recruitment and 
study progress, monitoring center performance, and data quality evaluation and interpretation; performed the statistical analyses; and reviewed and revised the manuscript. Mr. Kallan performed the statistical analyses and reviewed and revised the manuscript. Dr. Howell conceptualized the study design and protocol development and contributed to critical aspects of evaluation and analysis of data. Dr. Adzick conceptualized the study design and protocol development; contributed to critical aspects of the conduct of this research including assessment of patient recruitment, oversight of data quality, and evaluation and analysis of data; conceptualized the substudy; and preformed the critical revision of the manu- script. All authors approved the final manuscript as submitted and agree to be accountable for all aspects of the work. Comments and views of the authors do not necessarily represent views of the $\mathrm{Na}$ tional Institutes of Health.

\section{Clinical Trial Registration}

The trial has been registered at www.clinicaltrials.gov (identifier NCT 00060606).

\section{References}

1 Adzick NS, Thom EA, Spong CY, Brock JW, Burrows PK, Johnson MP, et al. A randomized trial of prenatal versus postnatal repair of myelomeningocele. N Engl J Med. 2011; 364(11):993-1004.

2 Tulipan N, Wellons JC 3rd, Thom EA, Gupta $\mathrm{N}$, Sutton LN, Burrows PK, et al. Prenatal surgery for myelomeningocele and the need for cerebrospinal fluid shunt placement. J Neurosurg Pediatr. 2015 Dec;16(6):613-20.

3 Farmer DL, Thom EA, Brock JW 3rd, Burrows $\mathrm{PK}$, Johnson MP, Howell LJ, et al. MOMS investigators. The Management of Myelomeningocele Study: full cohort 30 month pediatric outcomes. Am J Obstet Gynecol. 2017;9378(17):32475-4.

4 American College of Obstetricians and Gynecologists. ACOG committee opinion no. 550: maternal-fetal surgery for myelomeningocele. Obstet Gynecol. 2013;121:218-9.

5 Committee on Practice Bulletins-Obstetrics. Practice bulletin no. 187: neural tube defects. Obstet Gynecol. 2017 Dec;130(6):e279-90.

6 Wallingford JB, Niswander LA, Shaw GM, Finnell RH. The continuing challenge of understanding, preventing, and treating neural tube defects. Science. 2013 Mar 1;339(6123): 1222002.

7 Harrison MR. Fetal surgery. Am J Obstet Gynecol. 1996;174(4):1255-64.

8 Johnson MP, Bennett KA, Rand L, Burrows PK, Thom EA, Howell LJ, et al. The Management of Myelomeningocele Study: obstetrical outcomes and risk factors for obstetrical complications following prenatal surgery. Am J Obstet Gynecol. 2016;215(6):778.e1-e9.

9 Wu CS, Chen CM, Chou HC. Pulmonary hypoplasia induced by oligohydramnios: findings from animal models and a populationbased study. Pediatr Neonatol. 2017 Feb; 58(1):3-7.

10 Tita AT, Landon MB, Spong CY, Lai Y, Leveno KJ, Varner MW, et al. Timing of elective repeat cesarean delivery at term and neonatal outcomes. N Engl J Med. 2009;360(2):111-20.
11 Prefumo F, Ferrazzi E, Di Tommaso M, Severi FM, Locatelli A, Chirico G, et al. Neonatal morbidity after cesarean section before labor at $34(+0)$ to $38(+6)$ weeks: a cohort study. J Matern fetal Neonatal Med. 2016;29(8):13348.

12 Einchenwald E. Caffeine for apnea of prematurity. N Engl J Med. 2016;355(9):958-60.

13 Levin JC, Jang J, Rhein LM. Apnea in the otherwise healthy, term newborn: National Prevalence and Utilization during the birth hospitalization. J Pediatr. 2017;181:67-73.e1.

14 Bader D, Riskin A, Paz E, Kugelman A, Tirosh E. Breathing patterns in term infants delivered by caesarean section. Acta Paediatr. 2004;93(9):1216-20.

15 Allen MC. Outcome and follow-up of high risk infants. In: Taeusch HW, Ballard RA, editors. Avery's diseases of the newborn. Philadelphia: Saunders; 1998. p. 413-28.

16 Moldenhauer JS, Soni S, Rintoul NE, Spinner SS, Khalek N, Martinez-Poyer J, et al. Fetal myelomeningocele repair: the post-MOMS experience at the Children's Hospital of Philadelphia. Fetal Diagn Ther. 2015;37(3):23540.

17 Hamdan AH, Walsh W, Bruner JP, Tulipan $\mathrm{N}$. Intrauterine myelomeningocele repair: effect on short-term complications of prematurity. Fetal Diagn Ther. 2004;19(1):83-6.

18 Sarici SU, Serdar MA, Korkmaz A, Erdem G, Oran O, Tekinalp G, et al. Incidence, course, and prediction of hyperbilirubinemia in nearterm and term newborns. Pediatrics. 2004; 113(4):775-80.

19 Maisels MJ, Watchko JF, Bhutani VK, Stevenson DK. An approach to the management of hyperbilirubinemia in the preterm infant less than 35 weeks of gestation. J Perinatol. 2012; 32(9):660-4.

20 Blencowe H, Cousens S, Chou D, Oestergaard M, Say L, Moller AB, et al. Born too soon: the global epidemiology of 15 million preterm births. Reprod Health. 2013;10(Suppl 1):S2.
21 Vohr B. Long-term outcomes of moderately preterm, late preterm, and early term infants. Clin Perinatol. 2013;40(4):739-51.

22 Brock JW 3rd, Carr MC, Adzick NS, Burrows PK, Thomas JC, Thom EA, et al. Bladder function after fetal surgery for myelomeningocele. Pediatrics. 2015 Oct;136(4):e906-13.

23 Antiel RM, Flake AW, Johnson MP, Khalek N, Rintoul NE, Lantos JD, et al. Specialtybased variation in applying maternal-fetal surgery trial evidence. Fetal Diagn Ther. 2017; 42(3):210-7.

24 Antiel RM, Collura CA, Flake AW, Johnson MP, Rintoul NE, Lantos JD, et al. Physician views regarding the benefits and burdens of prenatal surgery for myelomeningocele. J Perinatol. 2017 Sep;37(9):994-8.

25 Antiel RM, Flake AW, Collura CA, Johnson MP, Rintoul NE, Lantos JD, et al. Weighing the social and ethical considerations of maternal-fetal surgery. Pediatrics. 2017 Dec;140(6): e20170608.

26 Soni S, Moldenhauer JS, Spinner SS, Rendon N, Khalek N, Martinez-Poyer J, et al. Chorioamniotic membrane separation and preterm premature rupture of membranes complicating in utero myelomeningocele repair. Am J Obstet Gynecol. 2016;214(5):647.e1-e7.

27 Elbabaa SK, Gildehaus AM, Pierson MJ, Albers JA, Vlastos EJ. First 60 fetal in-utero myelomeningocele repairs at Saint Louis Fetal Care Institute in the post-MOMS trial era: hydrocephalus treatment outcomes (endoscopic third ventriculostomy versus ventriculoperitoneal shunt). Childs Nerv Syst. 2017; 33(7):1157-68.

28 Bennett KA, Carroll MA, Shannon CN, Braun SA, Dabrowiak ME, Crum AK, et al. Reducing perinatal complications and preterm delivery for patients undergoing in utero closure of fetal myelomeningocele: further modifications to the multidisciplinary surgical technique. J Neurosurg Pediatr. 2014;14(1):108-14. 